\title{
Diseño y estandarización de la técnica de PCR para Porphyromonas gingivalis
}

Design and standardization of PCR technique

for Porphyromonas gingivalis
Concepção e padronização das PCR técnica

paraPorphyromonas gingivalis
Maria Rosenda Britos

Facultad de Odontología. Universidad Nacional del Nordeste. Corrientes, Argentina.

\section{Cynthya Solange Sin}

Biotecnología Microbiana para la Innovación Alimentaria (BiMIA) - (IMIT) Instituto de Modelado e Innovación Tecnológica.

CONICET - Argentina

Silvia Mercedes Ortega Facultad de Odontología. Universidad Nacional del Nordeste. Corrientes, Argentina

\section{Olga Miriam Vasek}

Biotecnología Microbiana para la Innovación Alimentaria (BiMIA) - (IMIT) Instituto de Modelado e Innovación Tecnológica. CONICET - Argentina

\section{Resumen}

El objetivo del presente trabajo fue diseñar y estandarizar la técnica de PCR para detección en líquido gingival de Porphyromonas gingivalis, en pacientes con enfermedad periodontal. Material y métodos: Se utilizaron iniciadores específicos para el gen ARNr I6s de Porphyromonas gingivalis. La especificidad de los iniciadores se ensayó utilizando material genético extraído de la cepa de referencia Porphyromonas gingivalis ATCC 33277. Se ajustaron las condiciones de amplificación y concentraciones de la mezcla de reacción. Para validar la técnica se aplicó a diez muestras clínicas de líquido gingival de pacientes con enfermedad periodontal. Resultados: Se vizualizaron bandas nítidas a 197pb utilizando cebadores específicos en seis muestras clínicas, y se obtuvo sensibilidad hasta 15 $\mathrm{ug} / \mathrm{ml}$ de $A D N$ purificado de la cepa de referencia ATCC 33277.Conclusiones: Se validó y estandarizó una PCR sencilla para la detección de Porphyromonas gingivalis en líquido gingival.

\section{Palabras claves}

PCR, Porphyromonas gingivalis, liquido gingival. 
REVISTA FACULTAD DE ODONTOLOGÍA

ISSN No 1668-7280 - Vol. X No 1 - 2017

26
INVESTIGACIÓN

Britos - Sin - Ortega - Vasek

\section{Abstract}

The aim of the present work was to design and standardize the PCR technique for the detection of Porphyromonas gingivalis in gingival fluid in patients with periodontal disease. Material and methods: Specific primers were used for the Porphyromonas gingivalis 16s rRNA gene. The specificity of the primers was assayed using genetic material extracted from the reference strain Porphyromonas gingivalis ATCC 33277. The amplification conditions and concentrations of the reaction mixture were adjusted. To validate the technique was applied to I0 clinical samples of gingival fluid of patients with periodontal disease. Results: Clear bands were visualized at 197bp using specific primers in 6 clinical samples, and sensitivity was obtained sensitivity up to I5ug / ml purified DNA from the reference strain ATCC 33277. Conclusions: A single PCR was validated and standardized for the detection of Porphyromonas Gingivalis in gingival fluid.

\section{Key words}

PCR, Porphyromonas gingivalis, gingival fluid.

\section{Resumo}

O objectivo deste estudo era conceber e padronizar a técnica de PCR para a detecção de fluido gengival Porphyromonas gingivalis em doentes com doença periodontal. foram utilizados iniciadores específicos para o gene de rRNA I6S de Porphyromonas gingivalis: Material e métodos. A especificidade dos iniciadores foi testada utilizando extraído a partir da estirpe de referência condições de amplificação do material genético ATCC 33277. Porphyromonas gingivalis e concentrações da mistura reaccional foi ajustado. Para validar a técnica foi aplicada para dez amostras clínicas de fluido gengival de pacientes com doença periodontal. Resultados: bandas nítidas são vizualizaron de 197pb utilizando iniciadores específicos em seis amostras clínicas e de sensibilidade foi obtida até $15 \mathrm{ug} / \mathrm{ml}$ de ADN purificado a partir da estirpe de referência ATCC 33277.Conclusiones: foi validado e estandardizado simples PCR para a detecção de Porphyromonas gingivalis em fluido gengival.
Palavras chave

PCR, Porphyromonas gingivalis, fluido gengival.

\section{Introducción}

La periodontitis es una enfermedad, que se caracteriza por la pérdida de inserción periodontal de las piezas dentarias. La periodontitis se considera una enfermedad infecciosa de origen poli-microbiano y la literatura menciona a más de 300 patógenos posiblemente relacionados con la destrucción periodontal'. En la variada microbiota subgingival, diferentes especies Gram negativas, anaerobias estrictas y microaerófilas juegan un papel primordial en el inicio y progresión de la enfermedad periodontal. Diferentes complejos microbianos se asociaron con la secuencia de colonización sobre la superficie de la pieza dentaria y con la gravedad de la enfermedad. El complejo rojo ${ }^{2}$, que aparece tardíamente en la secuencia del desarrollo del biofilm, comprende especies que se consideran microorganismos patógenos periodontales tales como Porphyromonas gingivalis (P.gingivalis), Treponema dentícola, y Tannerella forsythia ${ }^{1,3,4}$. Los expertos coinciden en que estas bacterias inician la periodontitis humana y la perpetúan. De hecho, en el Taller Mundial de 1999 sobre Periodoncia clínica se concluyó que la mayor parte de las periodontitis humanas son causadas por las especies antes mencionadas, a las que se suma Aggregatibacter (antes Actinobacillus) actinomycetemcomitans ${ }^{5}$.

P.gingivalis es un cocobacilo gramnegativo, anaerobio estricto, con factores de virulencia que le proveen un gran potencial para colonizar e invadir tejidos periodontales, modular la respuesta inmune del huésped, desencadenar una respuesta inflamatoria crónica y colaborar con los procesos de destrucción de tejido periodontal y hueso alveolar ${ }^{6-9}$. Conocer los meca nismos mediante los cuales $P$.gingivalis evade los ataques del sistema inmune, es de suma importancia para comprender su rol en la periodontitis. La interacción entre las células del huésped y $P$. gingivalis puede ser la clave para mantener la salud o la progresión de la enfermedad. Este microorganismo desempeña un papel crucial en la disbiosis dentro de la comunidad microbiana oral, quizás, al servir como un disruptor de comunicación en- 
REVISTA FACULTAD DE ODONTOLOGÍA ISSN No 1668-7280 - Vol. X No 1 - 2017

27
INVESTIGACIÓN

Diseño y estandarización de la técnica de

PCR para Porphyromonas gingivalis tre el biofilm poli-microbiano y el sistema inmune del huésped ${ }^{10}$. Se considera que las comunidades microbianas exhiben una virulencia sinérgica que les permite soportar la respuesta inmunológica del huésped $y$, también, desarrollar mecanismos para valerse del proceso inflamatorio, utilizando los tejidos deteriorados del huésped como nutrientes. Surgen, de este modo, nuevos modelos para entender los mecanismos y epidemiología de las infecciones poli-microbianas tales como la periodontitis, integrando la interacción microbiana $y$ elementos de la inmunidad inmune innata y adaptativa, que inician y propagan la inflamación periodontal crónica". Estas características permiten que se considere a $P$. gingivalis como el principal patógeno de la periodontitis y de enfermedades sistémicas como artritis reumatoidea y enfermedades cardiovasculares ${ }^{12}$. Teniendo en cuenta el rol que desempeña este microorganismo en la iniciación y progreso del proceso inflamatorio periodontal, su identificación se hace necesaria para definir la etiología de la enfermedad e instaurar un tratamiento adecuado. Tradicionalmente, la tipificación se basó en métodos de cultivo a partir de su aislamiento, incluyendo a la microscopía, los criterios fenotípicos y bioquímicos. Sin embargo, estas pruebas son laboriosas $y$, a veces, proporcionan resultados conflictivos, lo cual puede originarse en la variación existente entre cepas de una misma especie. Las técnicas basadas en el análisis de ADN se utilizan para identificar bacterias en forma directa a partir de muestras clínicas y eludir la necesidad de cultivo In Vitro ${ }^{13}$

En microbiología, esta metodología permite la detección de secuencias de ácidos nucleicos (ADN ○ ARN) que son específicos y conservados para cada microorganismo, a partir de diferentes matrices. Su aplicación, surge como una necesidad para detectar microorganismos de difícil crecimiento en cultivo tradicional o desarrollos tardíos. PCR es una de las herramientas de amplificación más sensible y que, en la actualidad, se aplica más satisfactoriamente en el área de microbiología clínica. Particularmente, para los microorganismos patógenos que son difíciles de aislar In Vitro o que presentan crecimiento lento, así como para el agente etiológico de aquellas infecciones clínicamente atribuibles a microorganismos emergentes ${ }^{14}$.
Para la detección universal bacteriana, ARN ribosomal $16 \mathrm{~S}$ es el utilizado ampliamente, debido a su omnipresencia en todos los organismos procariotas, con porciones conservadas a lo largo de la evolución y, a partir de la puesta en evidencia de porciones de su secuencia es posible obtener información filogenética y taxonómica ${ }^{15}$.

Desde su publicación, la técnica de PCR se utilizó ampliamente en Ciencias Médicas y de la salud tal como la Odontología ${ }^{16}$. La amplificación de secuencias de ácidos nucleicos mediante PCR para la detección de los distintos microorganismos periodontopatógenos se utilizó para agentes etiológicos de distintos tipos de enfermedad periodontal ${ }^{17,18}$.El objetivo del presente trabajo fue estandarizar y optimizar la técnica de PCR específica para la detección de Porphyromonas gingivalis en muestras de líquido gingival o crevicular.

\section{Materiales y Métodos}

Selección de los iniciadores: Para el diseño y optimización de la técnica de PCR se utilizaron iniciadores específicos para el gen que codifica una región conservada del ARNr I6S o ADN I6S (gen-housekeeping) en Porphyromonas gingivalis. La especificidad de los iniciadores se ensayó empleando material genético extraído de la cepa ATCC $®$ de Porphyromonas gingivalis $33277^{\mathrm{TM}}$. La estrategia del ensayo se diseñó en forma simple y económica. Se utilizaron los cebadores descriptos en el protocolo de PCR de acuerdo con Quintero y col (20II) ${ }^{17}$ cuya banda se visualiza a 197 pares de bases (pb). Extracción de ADN: Para la extracción de ADN, se tomó una alícuota de $100 \mu l$ de la suspensión celular y se resuspendió en solución fisiológica $(400 \mu \mathrm{l})$, luego se homogeneizó en vortex durante 20 segundos. Se centrifugó (4 min, 1.200 rpm), se desechó el sobrenadante y se recuperó el pellet celular para la extracción del material genético. El ADN se extrajo empleando el protocolo que emplea Bromuro de cetil trimetilamonio (CTAB) de acuerdo con lo sugerido por Stewart y col. (1993)19 y, posteriormente, se purificó con cloroformo-alcohol isoamílico. Para evaluar la cantidad y calidad de ADN genómi- 
co obtenido se utilizó el fotómetro UV AmpliQuat, AQ-07 Nucleic Acid se midió la absorbancia a $260 \mathrm{~nm}$ (A260) y $280 \mathrm{~nm}$ (A280) utilizando la fórmula siguiente: $[A D N]=A 260 \mathrm{~nm} \times \mathrm{D} \times$ $50 \mu \mathrm{g} / \mathrm{mL} \mathrm{D}=$ factor de dilución. El grado de pureza se calculó dividiendo la absorbancia a 260 $\mathrm{nm}$ entre la absorbancia a $280 \mathrm{~nm}$. Condiciones de PCR. Las secuencias de cebadores utilizadas para la identificaciónde $P$. gingivalis fueron $\mathrm{Pg}$ IF 5'-TGTAGATGACTGAAAACC-3' - Pg-2 R 5'-ACGTCATCCCCACCTTCCTC-3', que amplifican 197 pb. La reacción se realizó con un volumen final de $20 \mu \mathrm{l}$. Se realizaron curvas para obtener las concentraciones óptimas de $\mathrm{MgCl}_{2}$ y de oligonucleótidos. Las concentraciones finales fueron: IX buffer de PCR, $2 \mathrm{mM}$ de $\mathrm{MgCl}_{2}, 0,25$ mM de cada dNTP (Biodynamics, Argentina) I $\mu M$ de cada primer y I,0 U Taq DNA polimerasa (Promega, Argentina). Se empleó como control positivo la cepa de referencia $P$. gingivalis ATCC® $33277^{\mathrm{TM}}$ y como control negativo, agua. Programa de termociclado. La secuencia de ciclado (termociclador Bio-Rad, China) consistió en I ciclo de desnaturalización durante 5 min a $94{ }^{\circ} \mathrm{C}$, seguido de 40 ciclos a $94^{\circ} \mathrm{C}$ durante $30 \mathrm{~s}$, unión del cebador a $55^{\circ} \mathrm{C}$ durante 30 $\mathrm{s}$, extensión a $72{ }^{\circ} \mathrm{C}$ durante $45 \mathrm{~s}$ con extensión final a $72{ }^{\circ} \mathrm{C}$ durante 10 min e incubación adicional a $4{ }^{\circ} \mathrm{C}$. Análisis del producto de amplificación por electroforesis en gel. Los productos PCR se separaron por electroforesis en un gel de agarosa $1 \%$ en buffer TBEIX. Se sembraron $10 \mathrm{ml}$ en cada pocillo más $3 \mathrm{ml}$ de Gel Red 10,000X (Biotium, USA), diluido ( $3 X$ ) en agua destilada-deionizada más $3 \mathrm{ml}$ de buffer de carga $(60 \%$ glicerol, $0,05 \%$ azul bromofenol). Como marcador de Peso Molecular (PM) se utilizaron $5 \mu \mathrm{l}$ de CienMarker, (Biodynamics, Argentina). En una cuba horizontal Sub Cell@ GT (Biorad, China) y usando TBE IX (Trisma base, ácido bórico, EDTA, $\mathrm{pH} 8$ ) como buffer de corrida; se aplicaron 100 voltios durante $30 \mathrm{~min}$. Las bandas se visualizaron en un fotodocumentador (MaestroGen, Taiwan). Sensibilidad de la PCR. Se determinó empleando diluciones $1 / 10,1 / 100,1 / 1000$ del ADN purificado, obtenido a partir de la cepa de referencia $A T C C \circledR$ de $P$. gingivalis $33277^{\text {TM }}$. Para validar la técnica se procesaron diez muestras clínicas de pacientes que asistieron a las Clínicas Odontológicas de la Facultad de Odontología de la Universidad Nacional del Nordeste, con diagnóstico previo de periodontitis, cada participante firmo el consentimiento aprobado por el Comité de Bioética de la Facultad de Odontología de la UNNE. Las muestras fueron obtenidas con conos de papel absorbente (Asorbent Paper Points; Meta, Biomed, Chungbuk,Corea) conservadas en tubos Eppendorf a $-20^{\circ} \mathrm{C}$ hasta su procesamiento (Fig I).

\section{Resultados}

Con el método de extracción con CTAB y purificación se obtuvo $1552,65 \mathrm{ug} / \mathrm{ml}$ de DNA genómico de la cepa de referencia $A T C C \circledR P$. gingivalis 33277TM, y la calidad del DNA con una relación de OD260/OD280 de I,648 de pureza. Luego de estandarizar la metodología de PCR, se obtuvieron resultados satisfactorios con visualización de bandas nítidas a 197 $\mathrm{pb}$, correspondiente a los iniciadores específicos cuando se utilizó como molde ADN purificado correspondiente a la cepa de referencia ATCC $®$ P. gingivalis $33277^{\mathrm{TM}}$ de mientras que en las calles correspondientes al control negativo no se visualizaron bandas de amplificación . Para analizar la sensibilidad de la prueba se realizaron diluciones: $1 / 10,1 / 100$ y $1 / 1000$ de ADN genómico de la cepa de referencia $A T C C \circledR P$. gingivalis 33277TM, la prueba de PCR presento gran sensibilidad, llegando a detectar hasta 15 $\mathrm{ug} / \mathrm{ml}$ de $A D N$ purificado correspondiente a la dilución I//00 del templado. Cuando la técnica se aplicó a las muestras de líquido gingival se observó la presencia de banda compatible con $P$. gingivalis en seis muestras de pacientes con enfermedad periodontal, una banda nítida a 197 pb correspondiente al control positivo ADN purificado de la cepa de referencia $A T C C \circledR P$. gingivalis 33277TM y no se observó banda en el control negativo, donde de utilizo agua en reemplazo del templado. Fig I 


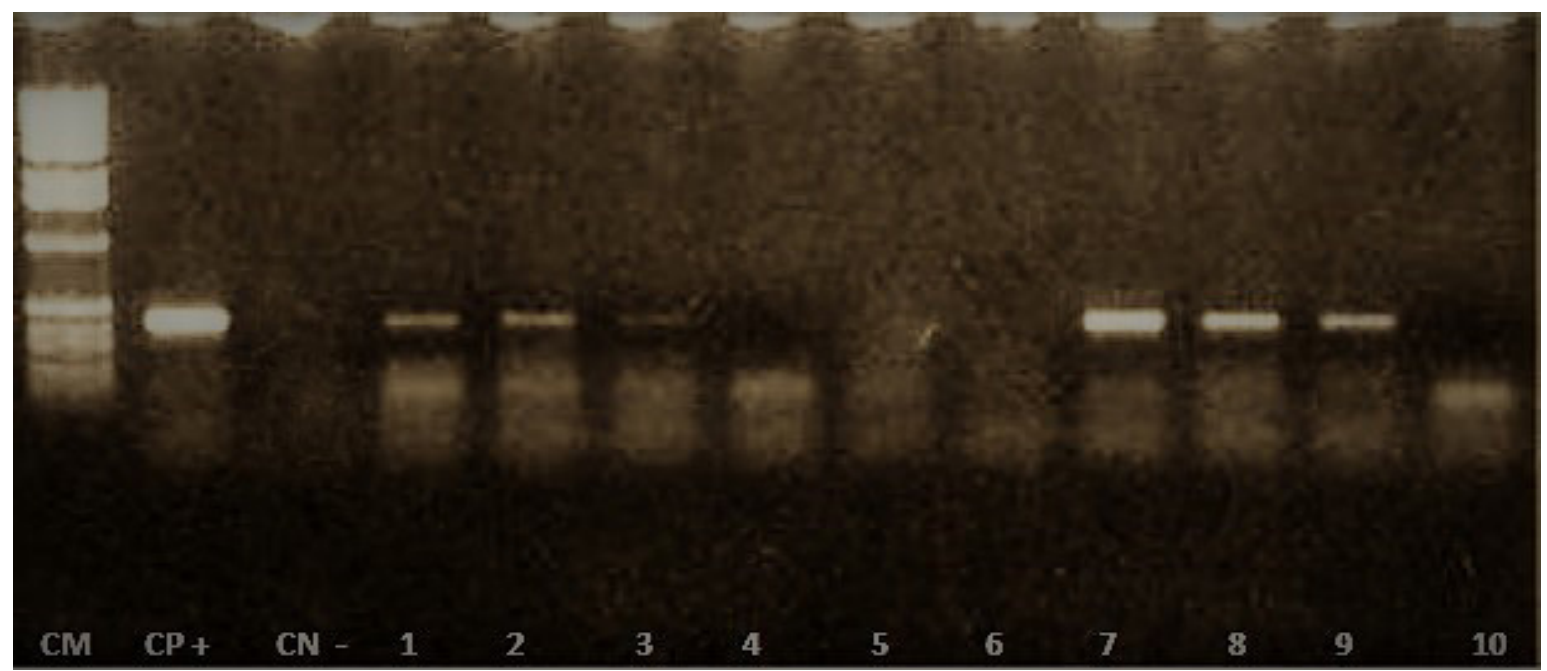

Figura I. Perfil electroforético de amplicones PCR especie específica de Porphyromonas gingivalis en agarosa (I\%). CM: marcador de peso molecular CP: Control positivo cepa de referencia Porphyromonas gingivalis ATCC $® 33277^{\mathrm{TM}} \mathrm{CN}$ : Control negativo. Calles I,2,3,7,8,9 muestras clínicas Porphyromonas gingivalis detectable. Calles 4,5,6 muestras clínicas Porphyromonas gingivalis no detectable.

\section{Discusión}

En la actualidad surgen nuevas líneas de investigación sobre la diversidad bacteriana dentro de las biopelículas, su patogenicidad y la susceptibilidad propia del paciente para producir una respuesta inmune dirigida a los patógenos periodontales invasores. Entre las técnicas moleculares, PCR, particularmente, es la metodología más empleada para el diagnóstico de los microorganismos debido a su elevada sensibilidad y especificidad, por lo que resulta una herramienta útil para este fin $2^{20,21}$. La capacidad para detectar e identificar secuencias de ácidos nucleicos provenientes de microorganismos permitió la detección de bacterias tanto cultivables como viables no cultivables, entre ellas P. gingivalis. Mättö y col (1998)22 demostraron la efectividad de la metodología PCR en la detección de P. gingivalis en saliva comparándola con técnicas de cultivo $y$, arribaron a la conclusión que la capacidad de detección mediante PCR fue superior a la que emplea cultivo. Okada y col en el año $2000^{22}$, recogieron material de placa a partir de cepillos dentales de niños, con el objetivo de detectar la presencia de Actinobacillus actinomycetemcomitans y $\mathrm{P}$. gingivalis utilizando PCR. Estos autores, indicaron que dichos microorganismos raramente se detectaron en las cavidades orales de niños sanos, a diferencia de aquellos que presentaron patologías. Sakamoto y col en el año $200 \mathrm{I}^{23}$, compararon las metodologías de PCR convencional, PCR en tiempo real utilizando el sistema LightCyclerTM y, el método de cultivo para detectar y cuantificar bacterias periodontopatógenas tales como A. actinomycetemcomitans, B. forsythus, P. gingivalis, T. dentícola y T. socranskii en saliva y placa subgingival. Tomazinho y col en $2007^{24}$ evaluó la prevalencia de P. gingivalis $y$ otros microorganismos en lesiones periapicales crónicas por metodología de cultivo y PCR hallando un $15.2 \%$ y $43.3 \%$ con cada método respectivamente. Este hallazgo confirmó los resultados reportados en estudios anteriores $^{21,25}$ que mostraron mayor sensibilidad de la técnica PCR que la de cultivo para la detección de bacilos anaerobios estrictos. El menor porcentaje de prevalencia empleando metodología de cultivo, se debería a las dificultades para recuperar estos microorganismos en función de su elevada sensibilidad frente al oxígeno. Varios estudios concluyeron al comparar la tecnología de PCR cuantitativa y el procedimiento de cultivo, que la elevada sensibilidad y especificidad de 
la PCR cuantitativa, justifican su uso en los estudios epidemiológicos y en el diagnóstico clínico de los pacientes periodontales ${ }^{26,27}$.

$P$. gingivalis, por sus fimbrias se adhiere a células del endotelio vascular simulando receptores plaquetarios, favoreciendo así el proceso aterogénico; por la capacidad de invadir células endoteliales, evade el sistema inmune y además produce alteraciones hemostáticas. De igual manera, las mujeres embarazadas que padezcan enfermedad periodontal estarán expuestas a riesgo de parto pre-término o bajo peso del niño al nacer, dependiendo de la magnitud de la respuesta inflamatoria lo que se refleja en el grado de destrucción periodontal. En el sistema respiratorio, ya sea por aspiración o por vía hematógena $P$. gingivalis es reconocida por las células de la inmunidad natural a través de receptores tipo Toll, activando la producción de sustancias proinflamatorias ${ }^{28}$.

\section{Conclusión}

En la medida en que la microbiología evolucionó, en la cavidad bucal se aislaron, aproximadamente, unas 400 especies bacterianas, de las cuales el $30 \%$, aún, no se pudieron cultivar. PCR es una herramienta muy útil en diversos campos de investigación y en Odontología. Entre muchas de sus aplicaciones, se puede utilizar para el diagnóstico de virus, parásitos y bacterias de difícil cultivo por poseer una alta especificidad, sensibilidad y seguridad, por ofrecer un diagnóstico confiable, más rápido y menos laborioso que los cultivos convencionales para este tipo de microorganismos. La metodología PCR para detectar P. gingivalis es de particular interés en el diagnóstico médico y odontológico.Se validó y estandarizó una PCR sencilla para la detección de Porphyromonas gingivalis en líquido gingival. Conflicto de Intereses

No existen conflictos de intereses

Agradecimientos

Fuente de financiamiento: Secretaria General de Ciencia y Técnica Universidad Nacional del Nordeste.

\section{Bibliografía}

I. Bascones A, Caballero A. Actinobacillus Actinomycetemcomitans y Porphyromonas Gingivalis como principales patógenos periodontales 2000;12:69-75. Disponible en: http://scielo.isciii.es/pdf/peri/v12n2/originall.pdf

2. Socransky SS, Haffajee AD, Cugini MA, Smith C, Kent $\mathrm{RL}$ Jr. Microbial complexes in subgingival plaque. J Clin Periodontol 1998; 25: 134-44. DOI/I0.1III/j.160005IX.1998.tb02419.x/abstract

3. Holt S, Ebersole J. Porphyromonas gingivalis, Treponema denticola, y Tannerella forsythia:el «complejo rojo», un prototipo de consorcio patógeno polibacteriano en la periodontitis Periodontology 2000 2005;12:72-I22. DOI: $10.1111 /$ j.16000757.2005.00 I I3.x

4. Kumawat, R. M., Ganvir, S. M., Hazarey, V. K., Qureshi, A., \& Purohit, H. J. Detection of Porphyromonas gingivalis and Treponema denticola in chronic and aggressive periodontitis patients: A comparative polymerase chain reaction study. Contemporary Clinical Dentistry 2016 ; 7: 48I.

5. Roy C, Kenneth S. The pathogenesis of human periodontitis: an introduction. Periodontology 2000 1997; I4: 9-II.

6. Ramos D, Moromi H, Martínez E. Porphyromonas gingivalis: Patógeno predominante en la periodontitis crónica.Odontol. Sanmarquina 20 I I; I4: 34-38 Disponible en : http://sisbib.unmsm.edu.pe/bvrevistas/odontologia/20I I_nl/pdf/al I.pdf

7. Moreno S, Contreras A. Factores de Virulencia de Porphyromonas gingivalis. Rev Fundac Juan Jose Carraro 2013; 37: 16-27. Disponible en: http://www.fundacioncarraro.org/descarga/revista37_art2.pdf

8. Mysak J, Podzimek S, Sommerova P, Lyuya-Mi Y, Bartova J, Janatova T, Prochazkova J, Duskova J. Porphyromonas gingivalis: Major Periodontopathic Pathogen Overview. J Immunol Research 2014 disponible en: http://dx.doi.org/ I0.I I55/20 I4/476068

9. Pandit N, Changela R, Bali D, Tikoo P, Gugnani S. Porphyromonas gingivalis : Its virulence and vaccine. J Int Clin Dent Res Organ 2015;7:5I.Disponible en: http:// www.jicdro.org/text.asp?20 I5/7/I/5 I/I53496

10. Cugini C, Klepac-Ceraj V, Rackaityte E, Riggs J, Davey M. Porphyromonas gingivalis: keeping the pathos out of the biont. J Oral Microbiol 2013; 5 Disponible en: http://dx.doi.org/I0.3402/jom.v5i0.19804

II. Hajishengallis G. Porphyromonas gingivalis-host interactions: open war or intelligent guerilla tactics? Microbes Infect 2009; I I: 637-45. Disponoble en: https:// www.ncbi.nlm.nih.gov/pmc/articles/PMC270425I/pdf/ nihms 103806.pdf

12. Burmistrz M, Dudek B, Staniec D, Rodriguez Martinez Jl, Bochtler M, Potempa J, Pyrc K. Functional analysis of Porphyromonas gingivalis W83 CRISPR-Cas syste ms.J.Bacteriol2015; 197:263 I-4I.Disponible en: http:// jb.asm.org/content/early/20 I5/05/| 9/JB.0026 I-I5.full. pdf+html 
REVISTA FACULTAD DE ODONTOLOGÍA

ISSN No 1668-7280 - Vol. X No 1 - 2017
INVESTIGACION

Diseño y estandarización de la técnica de

PCR para Porphyromonas gingivalis
13. Perea EJ. La flora de la boca en la era de la biología molecular. Med Oral Patol Oral Cir Bucal 2004; 9: I-I0. Disponible en: http://www.medicinaoral.com/ medoralfree0 I/v9Suppli/medoralv9supplip6.pdf

14. Ferreira Dos Santos C, Sakai V, Machado M, de Andrade Moreira A, Schippers D, Greene A. Reverse transcription and polymerase chain reaction: principles and applications in dentistry. J Appl Oral Sci 2004; I2: I- I I. Disponible en: http://www.scielo.br/pdf

15. Woese C. Bacterial Evolution. Microbiol Reviews 1987; 2: 22I-7I. Disponible en:http://www.ncbi. nlm.nih.gov/pmc/articles/PMC373/05/pdf/microrev00049-005I.pdf

16. Ledder RG, Gilbert P, Huws SA, Aarons L, Ashley MP, Hull PS. Molecular analysis of the subgingival microbiota in health and disease. Appl Environ Microbiol 2007; 73: 516-23. DOI: 10.1 I28/AEM.01419-06

17. Quintero AJ, Prada P, Inostroza CM, Chaparro A, Sanz AF, Ramírez VL, Morales HC. Presencia de Porphyromonas gingivalis, Tannerella forsythia, Treponema denticola y Aggregatibacter actinomycetemcomitans en el biofilm subgingival de pacientes diabéticos tipo 2: estudio transversal. Rev Clin Periodoncia Implantol Rehabil Oral 20I I; 4:54-58. Disponible en: www.scielo.cl/pdf/piro/v4n2/art03.pdf

18. Mayorga-Fayad I, Lafaurie G, Contreras A, Castillo D, Barón A, Aya M. Microflora subgingival en periodontitis crónica y agresiva en Bogotá, Colombia: un acercamiento epidemiológico Biomédica. Instituto Nacional de Salud Bogotá, Colombia2007;27: 21-33. Disponible en: http://www.scielo.org.co/scielo.php?script=sci_abs tract\&pid=SOI 20-4I 572007000100003

19. Stewart CN, Via LE. A rapid CTAB DNA isolation technique useful for RAPD fingerprinting and other PCR applications. BioTechniques 1993;14: 748-5I. Disponible en: https://es.scribd.com/doc/80909788/ARapid-CTAB-DNA-Isolation

20. Fenollar F, Raoult D. Molecular genetic methods for the diagnosis of fastidious microorganisms. APMIS 2004; I I2: 785-807. DOI: 10.1 I I I/j.1600-0463.2004. apm I I I I I-1206.x

21. Mättö J, Saarela M, Alaluusua S, Oja V, JousimiesSomer $\mathrm{H}$, Asikainen S. Detection of Porphyromonas gingivalis from saliva by PCR by using a simple sampleprocessing method. J Clin Microbiol 1998; 36: I57-60. Disponible en: http://pubmedcentralcanada.ca/pmcc/ articles/PMCI 24827/pdf/jm000 I57.pdf
22. Okada M, Hayashi F, Nagasaka N. Detection of Actinobacillus actinomycetemcomitans and Porphyromonas gingivalis in dental plaque samples from children 2 to 12 years of age. J Clin Periodontol 2000; 27 763-68. DOI: I0.1034/j.1600-05 Ix.2000.0270I0763.x

23. Sakamoto $M$, Takeuchi $Y$, Umeda M, Ishikawa I, Benno $Y$. Rapid detection and quantification of five periodontopathic bacteriaby real time PCR. [Abstract] Microbiol Immunol 200I; 45: 39-44. Disponible en: https:// www.ncbi.nlm.nih.gov/pubmed/I I 270605

24. Tomazinho L, Avila-Campos M. Detection of Porphyromonas gingivalis, Porphyromonas endodontalis, Prevotella intermedia, and Prevotella nigrescens in chronic endodontic infection. Oral Surgery, Oral Medicine, Oral Pathol, Oral Radiol and Endodontol 2007; 103: 285-88 Disponible en:http://www.sciencedirect. com/science/article/pii/SI 0792 I 040600375 I

25. Loesche W, Lopatin D, Stoll J, van Poperin N, Hujoel $\mathrm{P}$. Comparison of various detection methods for periodontopathic bacteria: can culture be considered the primary reference standard? J Clinl Microbiol 1992; 30:418-26. Disponible en: http://jcm.asm.org/ content/30/2/418.full.pdf

26. Boutaga $K$, van Winkelhoff $A$, Vandenbroucke-Grauls C, Savelkoul P. Comparison of Real-Time PCR and Culture for Detection of Porphyromonas gingivalis in Subgingival Plaque Samples. J Clin Microbiol 2003; 41: 4950-54. Disponible en : http://doi.org//0.1/28/ JCM.4I.II.4950-4954.2003

27. Lau L, Sanz M, Herrera D, Morillo JM, Martín C, Silva A. Quantitative real-time polymerase chain reaction versus culture: a comparison between two methods for the detection and quantification of Actinobacillus actinomycetemcomitans, Porphyromonas gingivalis and Tannerella forsythensis in subgingival plaque samples. J Clin Periodontol 2004; 31:106I-69. DOI: I0.1III/j.1600-05IX.2004.006I6.x

28. Orrego-Cardozo M, Parra-Gil MA, Salgado-Morales YP, Muñoz-Guarín E, Fandiño-Henao V. Porphyromonas gingivalis y enfermedades sistémicas. Rev. CES Odont 20I5; 28(I): 57-73 Disponible en: http:// revistas.ces.edu.co/index.php/odontologia/article/ view/3492/2385 